\title{
Differentiable Functions on Normed Linear Spaces $^{1}$
}

\author{
Yasunari Shidama \\ Shinshu University \\ Nagano, Japan
}

\begin{abstract}
Summary. In this article, we formalize differentiability of functions on normed linear spaces. Partial derivative, mean value theorem for vector-valued functions, continuous differentiability, etc. are formalized. As it is well known, there is no exact analog of the mean value theorem for vector-valued functions. However a certain type of generalization of the mean value theorem for vectorvalued functions is obtained as follows: If $\left\|f^{\prime}(x+t \cdot h)\right\|$ is bounded for $t$ between 0 and 1 by some constant $M$, then $\|f(x+t \cdot h)-f(x)\| \leq M \cdot\|h\|$. This theorem is called the mean value theorem for vector-valued functions. By this theorem, the relation between the (total) derivative and the partial derivatives of a function is derived [23].
\end{abstract}

MML identifier: NDIFF_5, version: $\underline{7.12 .01 \quad 4.167 .1133}$

The notation and terminology used here have been introduced in the following papers: [28], [29], [9], [4], [30], [12], [10], [25], [11], [1], [2], [26], [7], [3], [5], [8], $[17],[22],[20],[27],[21],[31],[14],[24],[18],[16],[15],[19],[13]$, and [6].

\section{Preliminaries}

In this paper $r$ is a real number and $S, T$ are non trivial real normed spaces. Next we state several propositions:

(1) Let $R$ be a function from $\mathbb{R}$ into $S$. Then $R$ is rest-like if and only if for every real number $r$ such that $r>0$ there exists a real number $d$ such that $d>0$ and for every real number $z$ such that $z \neq 0$ and $|z|<d$ holds $|z|^{-1} \cdot\left\|R_{z}\right\|<r$.

\footnotetext{
${ }^{1}$ This work was supported by JSPS KAKENHI 22300285.
}

(C) 2012 University of Białystok CC-BY-SA License ver. 3.0 or later ISSN $1426-2630(\mathrm{p}), 1898-9934(\mathrm{e})$ 
(2) Let $R$ be a rest of $S$. Suppose $R_{0}=0_{S}$. Let $e$ be a real number. Suppose $e>0$. Then there exists a real number $d$ such that $d>0$ and for every real number $h$ such that $|h|<d$ holds $\left\|R_{h}\right\| \leq e \cdot|h|$.

(3) For every rest $R$ of $S$ and for every bounded linear operator $L$ from $S$ into $T$ holds $L \cdot R$ is a rest of $T$.

(4) Let $R_{1}$ be a rest of $S$. Suppose $\left(R_{1}\right)_{0}=0_{S}$. Let $R_{2}$ be a rest of $S, T$. If $\left(R_{2}\right)_{0_{S}}=0_{T}$, then for every linear $L$ of $S$ holds $R_{2} \cdot\left(L+R_{1}\right)$ is a rest of $T$.

(5) Let $R_{1}$ be a rest of $S$. Suppose $\left(R_{1}\right)_{0}=0_{S}$. Let $R_{2}$ be a rest of $S, T$. Suppose $\left(R_{2}\right)_{0_{S}}=0_{T}$. Let $L_{1}$ be a linear of $S$ and $L_{2}$ be a bounded linear operator from $S$ into $T$. Then $L_{2} \cdot R_{1}+R_{2} \cdot\left(L_{1}+R_{1}\right)$ is a rest of $T$.

(6) Let $x_{0}$ be an element of $\mathbb{R}$ and $g$ be a partial function from $\mathbb{R}$ to the carrier of $S$. Suppose $g$ is differentiable in $x_{0}$. Let $f$ be a partial function from the carrier of $S$ to the carrier of $T$. Suppose $f$ is differentiable in $g_{x_{0}}$. Then $f \cdot g$ is differentiable in $x_{0}$ and $(f \cdot g)^{\prime}\left(x_{0}\right)=f^{\prime}\left(g_{x_{0}}\right)\left(g^{\prime}\left(x_{0}\right)\right)$.

(7) Let $S$ be a real normed space, $x_{1}$ be a finite sequence of elements of $S$, and $y_{1}$ be a finite sequence of elements of $\mathbb{R}$. Suppose len $x_{1}=\operatorname{len} y_{1}$ and for every element $i$ of $\mathbb{N}$ such that $i \in \operatorname{dom} x_{1}$ holds $y_{1}(i)=\left\|\left(x_{1}\right)_{i}\right\|$. Then $\left\|\sum x_{1}\right\| \leq \sum y_{1}$.

(8) Let $S$ be a real normed space, $x$ be a point of $S$, and $N_{1}, N_{2}$ be neighbourhoods of $x$. Then $N_{1} \cap N_{2}$ is a neighbourhood of $x$.

(9) For every non-empty finite sequence $X$ and for every set $x$ such that $x \in \prod X$ holds $x$ is a finite sequence.

Let $G$ be a real norm space sequence. One can verify that $\prod G$ is constituted finite sequences.

Let $G$ be a real linear space sequence, let $z$ be an element of $\prod \bar{G}$, and let $j$ be an element of $\operatorname{dom} G$. Then $z(j)$ is an element of $G(j)$.

One can prove the following propositions:

(10) The carrier of $\prod G=\prod \bar{G}$.

(11) Let $i$ be an element of $\operatorname{dom} G, r$ be a set, and $x$ be a function. If $r \in$ the carrier of $G(i)$ and $x \in \prod \bar{G}$, then $x+\cdot(i, r) \in$ the carrier of $\prod G$.

Let $G$ be a real norm space sequence. We say that $G$ is nontrivial if and only if:

(Def. 1) For every element $j$ of $\operatorname{dom} G$ holds $G(j)$ is non trivial.

Let us mention that there exists a real norm space sequence which is nontrivial.

Let $G$ be a nontrivial real norm space sequence and let $i$ be an element of $\operatorname{dom} G$. Note that $G(i)$ is non trivial.

Let $G$ be a nontrivial real norm space sequence. Note that $\prod G$ is non trivial.

The following propositions are true: 
(12) Let $G$ be a real norm space sequence, $p, q$ be points of $\prod G$, and $r_{0}, p_{0}$, $q_{0}$ be elements of $\prod \bar{G}$. Suppose $p=p_{0}$ and $q=q_{0}$. Then $p+q=r_{0}$ if and only if for every element $i$ of $\operatorname{dom} G$ holds $r_{0}(i)=p_{0}(i)+q_{0}(i)$.

(13) Let $G$ be a real norm space sequence, $p$ be a point of $\prod G, r$ be a real number, and $r_{0}, p_{0}$ be elements of $\prod \bar{G}$. Suppose $p=p_{0}$. Then $r \cdot p=r_{0}$ if and only if for every element $i$ of $\operatorname{dom} G$ holds $r_{0}(i)=r \cdot p_{0}(i)$.

(14) Let $G$ be a real norm space sequence and $p_{0}$ be an element of $\prod \bar{G}$. Then ${ }^{0} \prod_{G}=p_{0}$ if and only if for every element $i$ of $\operatorname{dom} G$ holds $p_{0}(i)=0_{G(i)}$.

(15) Let $G$ be a real norm space sequence, $p, q$ be points of $\prod G$, and $r_{0}, p_{0}$, $q_{0}$ be elements of $\prod \bar{G}$. Suppose $p=p_{0}$ and $q=q_{0}$. Then $p-q=r_{0}$ if and only if for every element $i$ of $\operatorname{dom} G$ holds $r_{0}(i)=p_{0}(i)-q_{0}(i)$.

\section{Mean Value Theorem for Vector-Valued Functions}

Let $S$ be a real linear space and let $p, q$ be points of $S$. The functor $] p, q[$ yielding a subset of $S$ is defined as follows:

(Def. 2) $] p, q[=\{p+t \cdot(q-p)$; $t$ ranges over real numbers: $0<t \wedge t<1\}$.

Let $S$ be a real linear space and let $p, q$ be points of $S$. We introduce $[p, q]$ as a synonym of $\mathcal{L}(p, q)$.

Next we state several propositions:

(16) For every real linear space $S$ and for all points $p, q$ of $S$ holds $] p, q[\subseteq$ $[p, q]$.

(17) Let $T$ be a non trivial real normed space and $R$ be a partial function from $\mathbb{R}$ to $T$. Suppose $R$ is total. Then $R$ is rest-like if and only if for every real number $r$ such that $r>0$ there exists a real number $d$ such that $d>0$ and for every real number $z$ such that $z \neq 0$ and $|z|<d$ holds $\frac{\left\|R_{z}\right\|}{|z|}<r$.

(18) Let $R$ be a function from $\mathbb{R}$ into $\mathbb{R}$. Then $R$ is rest-like if and only if for every real number $r$ such that $r>0$ there exists a real number $d$ such that $d>0$ and for every real number $z$ such that $z \neq 0$ and $|z|<d$ holds $\frac{|R(z)|}{|z|}<r$.

(19) Let $S, T$ be non trivial real normed spaces, $f$ be a partial function from $S$ to $T, p, q$ be points of $S$, and $M$ be a real number. Suppose that

(i) $[p, q] \subseteq \operatorname{dom} f$,

(ii) for every point $x$ of $S$ such that $x \in[p, q]$ holds $f$ is continuous in $x$,

(iii) for every point $x$ of $S$ such that $x \in] p, q[$ holds $f$ is differentiable in $x$, and

(iv) for every point $x$ of $S$ such that $x \in] p, q\left[\right.$ holds $\left\|f^{\prime}(x)\right\| \leq M$.

Then $\left\|f_{q}-f_{p}\right\| \leq M \cdot\|q-p\|$. 
(20) Let $S, T$ be non trivial real normed spaces, $f$ be a partial function from $S$ to $T, p, q$ be points of $S, M$ be a real number, and $L$ be a point of the real norm space of bounded linear operators from $S$ into $T$. Suppose that

(i) $[p, q] \subseteq \operatorname{dom} f$,

(ii) for every point $x$ of $S$ such that $x \in[p, q]$ holds $f$ is continuous in $x$,

(iii) for every point $x$ of $S$ such that $x \in] p, q$ [ holds $f$ is differentiable in $x$, and

(iv) for every point $x$ of $S$ such that $x \in] p, q\left[\right.$ holds $\left\|f^{\prime}(x)-L\right\| \leq M$. Then $\left\|f_{q}-f_{p}-L(q-p)\right\| \leq M \cdot\|q-p\|$.

\section{Partial Derivative of a Function of Several Variables}

Let $G$ be a real norm space sequence and let $i$ be an element of $\operatorname{dom} G$. The projection onto $i$ yielding a function from $\prod G$ into $G(i)$ is defined by:

(Def. 3) For every element $x$ of $\prod \bar{G}$ holds (the projection onto $\left.i\right)(x)=x(i)$.

Let $G$ be a real norm space sequence, let $i$ be an element of $\operatorname{dom} G$, and let $x$ be an element of $\prod G$. The functor $\operatorname{reproj}(i, x)$ yielding a function from $G(i)$ into $\prod G$ is defined by:

(Def. 4) For every element $r$ of $G(i)$ holds $(\operatorname{reproj}(i, x))(r)=x+\cdot(i, r)$.

Let $G$ be a nontrivial real norm space sequence and let $j$ be a set. Let us assume that $j \in \operatorname{dom} G$. The functor modetrans $(G, j)$ yields an element of $\operatorname{dom} G$ and is defined by:

(Def. 5) modetrans $(G, j)=j$.

Let $G$ be a nontrivial real norm space sequence, let $F$ be a non trivial real normed space, let $i$ be a set, let $f$ be a partial function from $\prod G$ to $F$, and let $x$ be an element of $\prod G$. We say that $f$ is partially differentiable in $x$ w.r.t. $i$ if and only if:

(Def. 6) $f \cdot \operatorname{reproj}(\operatorname{modetrans}(G, i), x)$ is differentiable in (the projection onto modetrans $(G, i))(x)$.

Let $G$ be a nontrivial real norm space sequence, let $F$ be a non trivial real normed space, let $i$ be a set, let $f$ be a partial function from $\prod G$ to $F$, and let $x$ be a point of $\prod G$. The functor partdiff $(f, x, i)$ yielding a point of the real norm space of bounded linear operators from $G$ (modetrans $(G, i))$ into $F$ is defined as follows:

(Def. 7) $\operatorname{partdiff}(f, x, i)=(f \cdot \operatorname{reproj}(\operatorname{modetrans}(G, i), x))^{\prime}(($ the projection onto modetrans $(G, i))(x))$. 


\section{Linearity of Partial Differential Operator}

For simplicity, we adopt the following rules: $G$ denotes a nontrivial real norm space sequence, $F$ denotes a non trivial real normed space, $i$ denotes an element of $\operatorname{dom} G, f, f_{1}, f_{2}$ denote partial functions from $\prod G$ to $F, x$ denotes a point of $\prod G$, and $X$ denotes a set.

Let $G$ be a nontrivial real norm space sequence, let $F$ be a non trivial real normed space, let $i$ be a set, let $f$ be a partial function from $\prod G$ to $F$, and let $X$ be a set. We say that $f$ is partially differentiable on $X$ w.r.t. $i$ if and only if:

(Def. 8) $X \subseteq \operatorname{dom} f$ and for every point $x$ of $\prod G$ such that $x \in X$ holds $f\lceil X$ is partially differentiable in $x$ w.r.t. $i$.

Next we state several propositions:

(21) For every element $x_{2}$ of $G(i)$ holds $\left\|\left(\operatorname{reproj}\left(i, 0 \prod_{G}\right)\right)\left(x_{2}\right)\right\|=\left\|x_{2}\right\|$.

(22) Let $G$ be a nontrivial real norm space sequence, $i$ be an element of $\operatorname{dom} G$, $x$ be a point of $\prod G$, and $r$ be a point of $G(i)$. Then $(\operatorname{reproj}(i, x))(r)-x=$ $\left(\operatorname{reproj}\left(i, 0 \prod_{G}\right)\right)(r-($ the projection onto $i)(x))$ and $x-(\operatorname{reproj}(i, x))(r)=$ $\left(\operatorname{reproj}\left(i, 0 \prod_{G}\right)\right)(($ the projection onto $i)(x)-r)$.

(23) Let $G$ be a nontrivial real norm space sequence, $i$ be an element of $\operatorname{dom} G$, $x$ be a point of $\prod G$, and $Z$ be a subset of $\prod G$. Suppose $Z$ is open and $x \in Z$. Then there exists a neighbourhood $N$ of (the projection onto $i)(x)$ such that for every point $z$ of $G(i)$ if $z \in N$, then $(\operatorname{reproj}(i, x))(z) \in Z$.

(24) Let $G$ be a nontrivial real norm space sequence, $T$ be a non trivial real normed space, $i$ be a set, $f$ be a partial function from $\prod G$ to $T$, and $Z$ be a subset of $\prod G$. Suppose $Z$ is open. Then $f$ is partially differentiable on $Z$ w.r.t. $i$ if and only if $Z \subseteq \operatorname{dom} f$ and for every point $x$ of $\prod G$ such that $x \in Z$ holds $f$ is partially differentiable in $x$ w.r.t. $i$.

(25) For every set $i$ such that $i \in \operatorname{dom} G$ and $f$ is partially differentiable on $X$ w.r.t. $i$ holds $X$ is a subset of $\prod G$.

Let $G$ be a nontrivial real norm space sequence, let $S$ be a non trivial real normed space, and let $i$ be a set. Let us assume that $i \in \operatorname{dom} G$. Let $f$ be a partial function from $\prod G$ to $S$ and let $X$ be a set. Let us assume that $f$ is partially differentiable on $X$ w.r.t. $i$. The functor $f \uparrow^{i} X$ yields a partial function from $\prod G$ to the real norm space of bounded linear operators from $G$ (modetrans $(G, i))$ into $S$ and is defined by:

(Def. 9) $\operatorname{dom}\left(f \uparrow^{i} X\right)=X$ and for every point $x$ of $\prod G$ such that $x \in X$ holds $\left(f \uparrow^{i} X\right)_{x}=\operatorname{partdiff}(f, x, i)$.

One can prove the following propositions:

(26) For every set $i$ such that $i \in \operatorname{dom} G$ holds $\left(f_{1}+f_{2}\right)$. $\operatorname{reproj}(\operatorname{modetrans}(G, i), x)=f_{1} \cdot \operatorname{reproj}(\operatorname{modetrans}(G, i), x)+f_{2}$. 
$\operatorname{reproj}(\operatorname{modetrans}(G, i), x)$ and $\left(f_{1}-f_{2}\right) \cdot \operatorname{reproj}(\operatorname{modetrans}(G, i), x)=$ $f_{1} \cdot \operatorname{reproj}(\operatorname{modetrans}(G, i), x)-f_{2} \cdot \operatorname{reproj}(\operatorname{modetrans}(G, i), x)$.

(27) For every set $i$ such that $i \in \operatorname{dom} G$ holds $r \cdot(f \cdot \operatorname{reproj}(\operatorname{modetrans}(G, i), x))=$ $(r \cdot f) \cdot \operatorname{reproj}(\operatorname{modetrans}(G, i), x)$.

(28) Let $i$ be a set. Suppose $i \in \operatorname{dom} G$ and $f_{1}$ is partially differentiable in $x$ w.r.t. $i$ and $f_{2}$ is partially differentiable in $x$ w.r.t. $i$. Then $f_{1}+f_{2}$ is partially differentiable in $x$ w.r.t. $i$ and partdiff $\left(f_{1}+f_{2}, x, i\right)=\operatorname{partdiff}\left(f_{1}, x, i\right)+$ $\operatorname{partdiff}\left(f_{2}, x, i\right)$.

(29) Let $i$ be a set. Suppose $i \in \operatorname{dom} G$ and $f_{1}$ is partially differentiable in $x$ w.r.t. $i$ and $f_{2}$ is partially differentiable in $x$ w.r.t. $i$. Then $f_{1}-f_{2}$ is partially differentiable in $x$ w.r.t. $i$ and partdiff $\left(f_{1}-f_{2}, x, i\right)=\operatorname{partdiff}\left(f_{1}, x, i\right)-$ $\operatorname{partdiff}\left(f_{2}, x, i\right)$.

(30) Let $i$ be a set. Suppose $i \in \operatorname{dom} G$ and $f$ is partially differentiable in $x$ w.r.t. $i$. Then $r \cdot f$ is partially differentiable in $x$ w.r.t. $i$ and $\operatorname{partdiff}(r$. $f, x, i)=r \cdot \operatorname{partdiff}(f, x, i)$.

\section{Continuous Differentiatibility of Partial Derivative}

Next we state the proposition

(31) $\|($ the projection onto $i)(x)\|\leq\| x \|$.

Let $G$ be a nontrivial real norm space sequence. One can verify that every point of $\prod G$ is len $G$-element.

We now state a number of propositions:

(32) Let $G$ be a nontrivial real norm space sequence, $T$ be a non trivial real normed space, $i$ be a set, $Z$ be a subset of $\prod G$, and $f$ be a partial function from $\prod G$ to $T$. Suppose $Z$ is open. Then $f$ is partially differentiable on $Z$ w.r.t. $i$ if and only if $Z \subseteq \operatorname{dom} f$ and for every point $x$ of $\prod G$ such that $x \in Z$ holds $f$ is partially differentiable in $x$ w.r.t. $i$.

(33) Let $i, j$ be elements of $\operatorname{dom} G, x$ be a point of $G(i)$, and $z$ be an element of $\prod \bar{G}$ such that $z=\left(\operatorname{reproj}\left(i, 0 \prod_{G}\right)\right)(x)$. Then

(i) if $i=j$, then $z(j)=x$, and

(ii) if $i \neq j$, then $z(j)=0_{G(j)}$.

(34) For all points $x, y$ of $G(i)$ holds $\left(\operatorname{reproj}\left(i, 0_{\Pi G}\right)\right)(x+y)=$ $\left(\operatorname{reproj}\left(i, 0_{\prod_{G}}\right)\right)(x)+\left(\operatorname{reproj}\left(i, 0 \prod_{G}\right)\right)(y)$.

(35) Let $x, y$ be points of $\prod G$. Then (the projection onto $\left.i\right)(x+y)=($ the projection onto $i)(x)+($ the projection onto $i)(y)$.

(36) For all points $x, y$ of $G(i)$ holds $\left(\operatorname{reproj}\left(i, 0_{\prod G}\right)\right)(x-y)=$ $\left(\operatorname{reproj}\left(i, 0 \prod_{G}\right)\right)(x)-\left(\operatorname{reproj}\left(i, 0 \prod_{G}\right)\right)(y)$. 
(37) Let $x, y$ be points of $\prod G$. Then (the projection onto $\left.i\right)(x-y)=($ the projection onto $i)(x)-($ the projection onto $i)(y)$.

(38) For every point $x$ of $G(i)$ such that $x \neq 0_{G(i)}$ holds $\left(\operatorname{reproj}\left(i, 0_{\prod G}\right)\right)(x) \neq$ ${ }^{0} \Pi^{\cdot}$

(39) For every point $x$ of $G(i)$ and for every element $a$ of $\mathbb{R}$ holds $\left(\operatorname{reproj}\left(i, 0 \prod_{G}\right)\right)(a \cdot x)=a \cdot\left(\operatorname{reproj}\left(i,{ } \prod_{G}\right)\right)(x)$.

(40) Let $x$ be a point of $\prod G$ and $a$ be an element of $\mathbb{R}$. Then (the projection onto $i)(a \cdot x)=a \cdot($ the projection onto $i)(x)$.

(41) Let $G$ be a nontrivial real norm space sequence, $S$ be a non trivial real normed space, $f$ be a partial function from $\prod G$ to $S, x$ be a point of $\Pi G$, and $i$ be a set. Suppose $f$ is differentiable in $x$. Then $f$ is partially differentiable in $x$ w.r.t. $i$ and $\operatorname{partdiff}(f, x, i)=f^{\prime}(x)$. $\operatorname{reproj}\left(\operatorname{modetrans}(G, i),{ }^{0} \prod_{G}\right)$.

(42) Let $S$ be a real normed space and $h, g$ be finite sequences of elements of $S$. Suppose len $h=\operatorname{len} g+1$ and for every natural number $i$ such that $i \in \operatorname{dom} g$ holds $g_{i}=h_{i}-h_{i+1}$. Then $h_{1}-h_{\text {len } h}=\sum g$.

(43) Let $G$ be a nontrivial real norm space sequence, $x, y$ be elements of $\prod \bar{G}$, and $Z$ be a set. Then $x+\cdot y\left\lceil Z\right.$ is an element of $\prod \bar{G}$.

(44) Let $G$ be a nontrivial real norm space sequence, $x, y$ be points of $\prod G$, $Z, x_{0}$ be elements of $\Pi \bar{G}$, and $X$ be a set. If $Z=0 \prod_{G}$ and $x_{0}=x$ and $y=Z+\cdot x_{0}\lceil X$, then $\|y\| \leq\|x\|$.

(45) Let $G$ be a nontrivial real norm space sequence, $S$ be a non trivial real normed space, $f$ be a partial function from $\prod G$ to $S$, and $x, y$ be points of $\prod G$. Then there exists a finite sequence $h$ of elements of $\prod G$ and there exists a finite sequence $g$ of elements of $S$ and there exist elements $Z, y_{0}$ of $\prod \bar{G}$ such that

$y_{0}=y$ and $Z=\prod_{\prod_{G}}$ and len $h=\operatorname{len} G+1$ and len $g=\operatorname{len} G$ and for every natural number $i$ such that $i \in \operatorname{dom} h$ holds $h_{i}=Z+\cdot y_{0}\left\lceil\operatorname{Seg}\left((\operatorname{len} G+1)-^{\prime}\right.\right.$ $i$ ) and for every natural number $i$ such that $i \in \operatorname{dom} g$ holds $g_{i}=f_{x+h_{i}}$ $f_{x+h_{i+1}}$ and for every natural number $i$ and for every point $h_{1}$ of $\prod G$ such that $i \in \operatorname{dom} h$ and $h_{i}=h_{1}$ holds $\left\|h_{1}\right\| \leq\|y\|$ and $f_{x+y}-f_{x}=\sum g$.

(46) Let $G$ be a nontrivial real norm space sequence, $i$ be an element of $\operatorname{dom} G$, $x, y$ be points of $\prod G$, and $x_{2}$ be a point of $G(i)$. If $y=(\operatorname{reproj}(i, x))\left(x_{2}\right)$, then (the projection onto $i)(y)=x_{2}$.

(47) Let $G$ be a nontrivial real norm space sequence, $i$ be an element of $\operatorname{dom} G$, $y$ be a point of $\prod G$, and $q$ be a point of $G(i)$. If $q=$ (the projection onto $i)(y)$, then $y=(\operatorname{reproj}(i, y))(q)$.

(48) Let $G$ be a nontrivial real norm space sequence, $i$ be an element of $\operatorname{dom} G$, $x, y$ be points of $\prod G$, and $x_{2}$ be a point of $G(i)$. If $y=(\operatorname{reproj}(i, x))\left(x_{2}\right)$, then $\operatorname{reproj}(i, x)=\operatorname{reproj}(i, y)$. 
(49) Let $G$ be a nontrivial real norm space sequence, $i, j$ be elements of $\operatorname{dom} G, x, y$ be points of $\prod G$, and $x_{2}$ be a point of $G(i)$. Suppose $y=(\operatorname{reproj}(i, x))\left(x_{2}\right)$ and $i \neq j$. Then (the projection onto $\left.j\right)(x)=($ the projection onto $j)(y)$.

(50) Let $G$ be a nontrivial real norm space sequence, $F$ be a non trivial real normed space, $i$ be an element of $\operatorname{dom} G, x$ be a point of $\prod G, x_{2}$ be a point of $G(i), f$ be a partial function from $\prod G$ to $F$, and $g$ be a partial function from $G(i)$ to $F$. If (the projection onto $i)(x)=x_{2}$ and $g=f \cdot \operatorname{reproj}(i, x)$, then $g^{\prime}\left(x_{2}\right)=\operatorname{partdiff}(f, x, i)$.

(51) Let $G$ be a nontrivial real norm space sequence, $F$ be a non trivial real normed space, $f$ be a partial function from $\prod G$ to $F, x$ be a point of $\prod G, i$ be a set, $M$ be a real number, $L$ be a point of the real norm space of bounded linear operators from $G$ (modetrans $(G, i))$ into $F$, and $p, q$ be points of $G$ (modetrans $(G, i))$. Suppose that

(i) $\quad i \in \operatorname{dom} G$,

(ii) for every point $h$ of $G$ (modetrans $(G, i))$ such that $h \in] p, q[$ holds $\|\operatorname{partdiff}(f,(\operatorname{reproj}(\operatorname{modetrans}(G, i), x))(h), i)-L\| \leq M$,

(iii) for every point $h$ of $G$ (modetrans $(G, i))$ such that $h \in[p, q]$ holds $(\operatorname{reproj}(\operatorname{modetrans}(G, i), x))(h) \in \operatorname{dom} f$, and

(iv) for every point $h$ of $G$ (modetrans $(G, i))$ such that $h \in[p, q]$ holds $f$ is partially differentiable in $(\operatorname{reproj}(\operatorname{modetrans}(G, i), x))(h)$ w.r.t. $i$.

Then $\left\|f_{(\operatorname{reproj}(\operatorname{modetrans}(G, i), x))(q)}-f_{(\operatorname{reproj}(\operatorname{modetrans}(G, i), x))(p)}-L(q-p)\right\| \leq$ $M \cdot\|q-p\|$.

(52) Let $G$ be a nontrivial real norm space sequence, $x, y, z, w$ be points of $\prod G, i$ be an element of $\operatorname{dom} G, d$ be a real number, and $p, q, r$ be points of $G(i)$. Suppose $\|y-x\|<d$ and $\|z-x\|<d$ and $p=$ (the projection onto $i)(y)$ and $z=(\operatorname{reproj}(i, y))(q)$ and $r \in[p, q]$ and $w=(\operatorname{reproj}(i, y))(r)$. Then $\|w-x\|<d$.

(53) Let $G$ be a nontrivial real norm space sequence, $S$ be a non trivial real normed space, $f$ be a partial function from $\prod G$ to $S, X$ be a subset of $\prod G$, $x, y, z$ be points of $\prod G, i$ be a set, $p, q$ be points of $G(\operatorname{modetrans}(G, i))$, and $d, r$ be real numbers. Suppose that $i \in \operatorname{dom} G$ and $X$ is open and $x \in$ $X$ and $\|y-x\|<d$ and $\|z-x\|<d$ and $X \subseteq \operatorname{dom} f$ and for every point $x$ of $\prod G$ such that $x \in X$ holds $f$ is partially differentiable in $x$ w.r.t. $i$ and for every point $z$ of $\prod G$ such that $\|z-x\|<d$ holds $z \in X$ and for every point $z$ of $\prod G$ such that $\|z-x\|<d$ holds $\|\operatorname{partdiff}(f, z, i)-\operatorname{partdiff}(f, x, i)\| \leq$ $r$ and $z=(\operatorname{reproj}(\operatorname{modetrans}(G, i), y))(p)$ and $q=($ the projection onto modetrans $(G, i))(y)$. Then $\left\|f_{z}-f_{y}-(\operatorname{partdiff}(f, x, i))(p-q)\right\| \leq\|p-q\| \cdot r$.

(54) Let $G$ be a nontrivial real norm space sequence, $h$ be a finite sequence of elements of $\prod G, y, x$ be points of $\prod G, y_{0}, Z$ be elements of $\prod \bar{G}$, and $j$ be an element of $\mathbb{N}$. Suppose $y=y_{0}$ and $Z={ }^{0} \prod_{G}$ and 
len $h=\operatorname{len} G+1$ and $1 \leq j \leq \operatorname{len} G$ and for every natural number $i$ such that $i \in \operatorname{dom} h$ holds $h_{i}=Z+\cdot y_{0}\left\lceil\operatorname{Seg}\left((\operatorname{len} G+1)-^{\prime} i\right)\right.$. Then $x+h_{j}=\left(\operatorname{reproj}\left(\operatorname{modetrans}\left(G,(\operatorname{len} G+1)-^{\prime} j\right), x+h_{j+1}\right)\right)(($ the projection onto modetrans $(G$, (len $\left.\left.\left.G+1)-{ }^{\prime} j\right)\right)(x+y)\right)$.

(55) Let $G$ be a nontrivial real norm space sequence, $h$ be a finite sequence of elements of $\prod G, y, x$ be points of $\prod G, y_{0}, Z$ be elements of $\prod \bar{G}$, and $j$ be an element of $\mathbb{N}$. Suppose $y=y_{0}$ and $Z={ }^{0} \prod G$ and len $h=\operatorname{len} G+1$ and $1 \leq j \leq \operatorname{len} G$ and for every natural number $i$ such that $i \in \operatorname{dom} h$ holds $h_{i}=Z+\cdot y_{0}\left\lceil\operatorname{Seg}\left((\operatorname{len} G+1)-^{\prime} i\right)\right.$. Then (the projection onto modetrans $\left(G,(\right.$ len $\left.\left.G+1)-{ }^{\prime} j\right)\right)(x+y)-($ the projection onto modetrans $\left.\left(G,(\operatorname{len} G+1)-^{\prime} j\right)\right)\left(x+h_{j+1}\right)=$ (the projection onto $\left.\operatorname{modetrans}\left(G,(\operatorname{len} G+1)-^{\prime} j\right)\right)(y)$.

(56) Let $G$ be a nontrivial real norm space sequence, $S$ be a non trivial real normed space, $f$ be a partial function from $\prod G$ to $S, X$ be a subset of $\prod G$, and $x$ be a point of $\prod G$. Suppose that

(i) $X$ is open,

(ii) $\quad x \in X$, and

(iii) for every set $i$ such that $i \in \operatorname{dom} G$ holds $f$ is partially differentiable on $X$ w.r.t. $i$ and $f \uparrow^{i} X$ is continuous on $X$.

Then

(iv) $\quad f$ is differentiable in $x$, and

(v) for every point $h$ of $\prod G$ there exists a finite sequence $w$ of elements of $S$ such that $\operatorname{dom} w=\operatorname{dom} G$ and for every set $i$ such that $i \in \operatorname{dom} G$ holds $w(i)=(\operatorname{partdiff}(f, x, i))(($ the projection onto modetrans $(G, i))(h))$ and $f^{\prime}(x)(h)=\sum w$.

(57) Let $G$ be a nontrivial real norm space sequence, $F$ be a non trivial real normed space, $f$ be a partial function from $\prod G$ to $F$, and $X$ be a subset of $\prod G$. Suppose $X$ is open. Then for every set $i$ such that $i \in \operatorname{dom} G$ holds $f$ is partially differentiable on $X$ w.r.t. $i$ and $f \uparrow^{i} X$ is continuous on $X$ if and only if $f$ is differentiable on $X$ and $f_{\uparrow X}^{\prime}$ is continuous on $X$.

\section{REFERENCES}

[1] Grzegorz Bancerek. Cardinal numbers. Formalized Mathematics, 1(2):377-382, 1990.

[2] Grzegorz Bancerek. The fundamental properties of natural numbers. Formalized Mathematics, 1(1):41-46, 1990.

[3] Grzegorz Bancerek. König's theorem. Formalized Mathematics, 1(3):589-593, 1990.

[4] Grzegorz Bancerek. The ordinal numbers. Formalized Mathematics, 1(1):91-96, 1990.

[5] Grzegorz Bancerek and Krzysztof Hryniewiecki. Segments of natural numbers and finite sequences. Formalized Mathematics, 1(1):107-114, 1990.

[6] Grzegorz Bancerek and Andrzej Trybulec. Miscellaneous facts about functions. Formalized Mathematics, 5(4):485-492, 1996.

[7] Czesław Byliński. The complex numbers. Formalized Mathematics, 1(3):507-513, 1990.

[8] Czesław Byliński. Finite sequences and tuples of elements of a non-empty sets. Formalized Mathematics, 1(3):529-536, 1990. 
[9] Czesław Byliński. Functions and their basic properties. Formalized Mathematics, 1(1):5565, 1990.

[10] Czesław Byliński. Functions from a set to a set. Formalized Mathematics, 1(1):153-164, 1990.

[11] Czesław Byliński. The modification of a function by a function and the iteration of the composition of a function. Formalized Mathematics, 1(3):521-527, 1990.

[12] Czesław Byliński. Partial functions. Formalized Mathematics, 1(2):357-367, 1990.

[13] Czesław Byliński. Introduction to real linear topological spaces. Formalized Mathematics, 13(1):99-107, 2005.

[14] Agata Darmochwał. The Euclidean space. Formalized Mathematics, 2(4):599-603, 1991.

[15] Noboru Endou, Yasunari Shidama, and Keiichi Miyajima. The product space of real normed spaces and its properties. Formalized Mathematics, 15(3):81-85, 2007, doi:10.2478/v10037-007-0010-y.

[16] Hiroshi Imura, Morishige Kimura, and Yasunari Shidama. The differentiable functions on normed linear spaces. Formalized Mathematics, 12(3):321-327, 2004.

[17] Jarosław Kotowicz. Real sequences and basic operations on them. Formalized Mathematics, 1(2):269-272, 1990.

[18] Anna Lango and Grzegorz Bancerek. Product of families of groups and vector spaces. Formalized Mathematics, 3(2):235-240, 1992.

[19] Hiroyuki Okazaki, Noboru Endou, Keiko Narita, and Yasunari Shidama. Differentiable functions into real normed spaces. Formalized Mathematics, 19(2):69-72, 2011, doi: 10.2478/v10037-011-0012-7.

[20] Beata Padlewska and Agata Darmochwał. Topological spaces and continuous functions. Formalized Mathematics, 1(1):223-230, 1990.

[21] Jan Popiołek. Real normed space. Formalized Mathematics, 2(1):111-115, 1991.

[22] Konrad Raczkowski and Paweł Sadowski. Real function differentiability. Formalized Mathematics, 1(4):797-801, 1990.

[23] Laurent Schwartz. Cours d'analyse, vol. 1. Hermann Paris, 1967.

[24] Yasunari Shidama. Banach space of bounded linear operators. Formalized Mathematics, 12(1):39-48, 2004.

[25] Andrzej Trybulec. Binary operations applied to functions. Formalized Mathematics, 1(2):329-334, 1990.

[26] Andrzej Trybulec. On the sets inhabited by numbers. Formalized Mathematics, 11(4):341$347,2003$.

[27] Wojciech A. Trybulec. Vectors in real linear space. Formalized Mathematics, 1(2):291-296, 1990.

[28] Zinaida Trybulec. Properties of subsets. Formalized Mathematics, 1(1):67-71, 1990.

[29] Edmund Woronowicz. Relations and their basic properties. Formalized Mathematics, 1(1):73-83, 1990.

[30] Edmund Woronowicz. Relations defined on sets. Formalized Mathematics, 1(1):181-186, 1990.

[31] Hiroshi Yamazaki and Yasunari Shidama. Algebra of vector functions. Formalized Mathematics, 3(2):171-175, 1992.

Received June 2, 2011 